\title{
Erratum to: Pneumococcal vaccination: what have we learnt so far and what can we expect in the future?
}

\author{
A. Torres $\cdot$ P. Bonanni $\cdot$ W. Hryniewicz $・$ M. Moutschen $\cdot$ \\ R. R. Reinert • T. Welte
}

Published online: 18 October 2014

(C) Springer-Verlag Berlin Heidelberg 2014

\section{Erratum to: Eur J Clin Microbiol Infect Dis \\ DOI 10.1007/s10096-014-2208-6}

The original version of this article unfortunately contains an error in Table 2. The pneumococcal vaccination recommendations for Ireland are incorrectly shown in Table 2. These recommendations have been corrected in the table below. The authors wish to apologize for these errors.

The online version of the original article can be found at http://dx.doi.org/ 10.1007/s10096-014-2208-6.

A. Torres $(\bowtie)$

Servei de Pneumologia, Hospital Clínic de Barcelona, Institut d'Investigacions Biomèdiques August Pi i Sunyer (IDIBAPS), CIBER de Enfermedades Respiratorias (CIBERes), University of Barcelona, Barcelona, Spain

e-mail: ATORRES@clinic.ub.es

P. Bonanni

Department of Health Sciences, University of Florence, Florence, Italy

W. Hryniewicz

National Medicines Institute, Warsaw, Poland

M. Moutschen

Department of Infectious Diseases and General Internal Medicine, $\mathrm{CHU}$ de Liège/University of Liège, Liège, Belgium

R. R. Reinert

Pfizer Vaccines (Medical Development Group and Scientific

Affairs), Paris, France

T. Welte

Klinic für Pneumologie, Medizinische Hochschule Hannover,

Hannover, Germany 
Table 2 National adult pneumococcal vaccination recommendations in Western Europe (Ireland)

\begin{tabular}{|c|c|c|c|}
\hline \multirow[b]{2}{*}{ Country (Date) $)^{\mathrm{a}}$} & \multirow[b]{2}{*}{ Vaccine } & \multicolumn{2}{|c|}{ Pneumococcal vaccine recommendation } \\
\hline & & Age based & At-risk based, with definition of risk \\
\hline $\begin{array}{l}\text { Ireland } \\
\text { (2013) [94] }\end{array}$ & PCV13/PPV23 & No & $\begin{array}{l}\text { High-risk group (18-64 years): Asplenia, hyposplenia (including splenectomy, } \\
\text { sickle cell disease, hemoglobinopathies, and coeliac disease); cochlear implant } \\
\text { (candidates, recipients); complement deficiency (particularly C1-C4); CSF leak } \\
\text { (congenital, complicating skull fracture, neurosurgery); immunosuppressive } \\
\text { conditions (e.g. some B- and T-cell disorders, HIV infection, leukaemia, } \\
\text { lymphoma) and those receiving immunosuppressive therapies; intracranial } \\
\text { shunt; post-haematopoietic stem-cell transplant; solid organ transplant }\end{array}$ \\
\hline Ireland (2013) [94] & PPV23 & $\geq 65$ years & $\begin{array}{l}\text { Medium-risk group (18-64 years): chronic heart, lung, or liver disease; chronic } \\
\text { renal disease or nephrotic syndrome; diabetes mellitus requiring insulin or oral } \\
\text { hypoglycaemic drugs; individuals with occupational exposure to metal fumes } \\
\text { (i.e. welders); smokers and alcoholics }\end{array}$ \\
\hline
\end{tabular}

CSF, cerebrospinal fluid; CNS, central nervous system; COPD, chronic obstructive pulmonary disease; HIV, human immunodeficiency virus; IPD, invasive pneumococcal disease; PCV, pneumococcal conjugate vaccine; PPV, pneumococcal polysaccharide vaccine; SCID, severe combined immunodeficiency

${ }^{\mathrm{a}}$ Date of implementation of recommendation

\section{Reference}

94. National Immunisation Advisory Committee of the Royal College of Physicians of Ireland (2013) Immunisation
Guidelines for Ireland, 2013 Edition (updated June 2014). Chapter 16: pneumococcal infection. Health Service Executive. Available online at: http://www.immunisation.ie/en/ HealthcareProfessionals/ImmunisationGuidelines/. Accessed 12 August 2014 